\title{
E1 humor como regulador de la actividad de imagen en el debate parlamentario
}

\author{
Humor as a regulatory face work mechanism within the parliamentary debate
}

\author{
Alejandro Romero Nieto
}

\begin{abstract}
Resumen
En este trabajo se presenta el análisis del funcionamiento del humor dentro de un género discursivo fuertemente reglado, como es el debate parlamentario, desde un punto de vista interaccional. En concreto, se analizan las sesiones plenarias que tuvieron lugar en la cámara regional de diputados de la Comunidad de Madrid, la Asamblea de Madrid, entre los años 2010 y 2011. El análisis se centra en aspectos como la cortesía y la actividad de imagen, entendida esta como el ethos que el político proyecta hacia su audiencia, en su doble faceta de cargo público y persona privada, y se hace especial hincapié en la manera en que las burlas inciden sobre la imagen del hablante: bien aumentándola, al presentarle como una persona ingeniosa y carismática, o bien reforzándola, al poner en evidencia los lazos de connivencia que existen entre él y los integrantes de su partido. La conclusión que extraemos es que el humor permite al hablante parlamentario mantener sus críticas dentro de los límites de lo democráticamente aceptable y, al mismo tiempo, efectuar una operación de autovalorización de la imagen, siempre con la intención de disminuir la imagen de sus adversarios políticos.
\end{abstract}

Palabras clave: discurso político, humor, imagen, ethos, análisis del discurso, debate parlamentario.

\begin{abstract}
This paper aims to present an interactional analysis of the functioning of humour that occurs within a strongly regulated genre, such as parliamentary debates. In particular, the sessions, which took place in the regional chamber of Madrid, the Assembly of Madrid, between the years 2010 and 2011. The focus is set on features such as politeness and, specially, facework, which is seen as the politicians' ethos project before their audience, both as public office and as private persons. Likewise, the analysis emphasises specifically on the way that mockery affects the speaker's self-face: both either increasing it, by means of appearing as a witty person, or reinforcing it, by means of revealing the bonds of connivance, which may exist between him and the members of his party. The conclusion that can be extracted is that humour allows parliamentarians to keep their criticism within the boundaries of what is democratically acceptable, and at the same time to perform a self-assessment operation, with the intention of decreasing the face of their adversaries.
\end{abstract}

Keywords: political discourse, humour, face-work, ethos, discourse analysis, parliamentary debate.

Alejandro Romero Nieto, Universidad Complutense de Madrid, aromeronieto83@gmail.com

Recibido: diciembre 2016 / Aceptado: abril 2017

DOI 10.17710/tep.2017.3.2.4romeronieto 


\section{Introducción}

El estudio del discurso político ha gozado de gran interés en los estudios de lingüística, especialmente en el ámbito de la pragmática y el análisis del discurso. Los primeros trabajos en este sentido se remontan a los años 80 del siglo XX, con los trabajos pioneros y sugerentes de Beattie (1982) o Atkinson (1988). En la década siguiente, son ya numerosos los lingüistas que, desde teorías y conceptos pragmáticos y desde el análisis del discurso, se ocupan de describir las características del discurso político, como Robin Lakoff (1990), Wilson (1990) o Connolly (1993).

En las últimas décadas, el estudio del discurso político se ha especializado, a través de la aportación de trabajos específicos, destinados a analizar determinados aspectos del discurso político en general (Chilton, 2004; Charaudeau, 2005; George Lakoff, 2007) o parlamentario en particular (Tsakona, 2009; Archakis y Tsakona, 2011; Tsakona, 2013; Saftoiu y Popescu, 2014).

En la investigación lingüística en España, el discurso político también ha despertado el interés de los analistas, en particular del discurso oral. En este sentido destacan trabajos relativos a aspectos como la cortesía, la prosodia o la gestualidad en los debates electorales (Blas Arroyo, 2001; González Sanz, 2014; Hidalgo Downing y Nieto y Otero, 2014), junto a otros más específicos sobre la estructura de los debates parlamentarios (Cortés Rodríguez, 2011; Bañón Hernández et al., 2012; Cortés Rodríguez, 2015); el valor de la ironía (Alcaide Lara, 2004, 2011); las funciones de la interrupción con valor humorístico como instrumento gestor de las relaciones de poder entre los diputados y la presidencia (Romero Nieto, 2017) o el valor de la gestualidad y la prosodia como marcadores del tono humorístico en el debate parlamentario (Romero Nieto, 2018)

En cuanto al humor, ha gozado también de interés en el ámbito de la lingüística, y se ha convertido en una fructífera área de estudio dentro de las teorías semánticas y pragmáticas, principalmente: Norrick (1993), Attardo (1994), Charaudeau (2013) en el ámbito internacional; y Yus (2003) o Ruiz Gurillo (2012) en el ámbito español han contribuido sobremanera a configurar un marco teórico lo suficientemente sólido como para abordar de forma adecuada el estudio del humor dentro de los campos de la fonología, la sociolingüística, la psicología y la pragmática.

Mientras que el humor goza de gran vitalidad como objeto de estudio, y el discurso político también, la unión de los dos ámbitos no ha recibido apenas atención (Tsakona, 2009; Archakis y Tsakona, 2011; Tsakona, 2013; Saftoiu y Popescu, 2014). Constituye, por

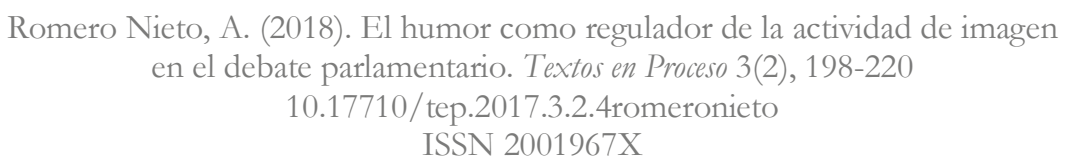


tanto, una parcela que queda por explorar, y que plantea este trabajo de investigación: es decir, el estudio del humor en el discurso político, un ámbito fundamentalmente serio a todos los efectos. En este contexto serio y fuertemente restringido, en donde el político está siendo evaluado continuamente tanto por sus adversarios como por sus potenciales votantes, queremos saber si el humor puede producirse y, en dicho caso, cuáles son los efectos y finalidades derivados de su uso. En un contexto como el político, de convenciones establecidas y muchas veces rígidas, el humor podría suponer un riesgo, un peligro para el político.

El interés de la investigación que propone este trabajo obedece a la intención de averiguar si el humor, que supone un riesgo para la imagen del hablante, es funcional dentro de un contexto de extrema seriedad como el debate parlamentario, donde la imagen del político, en tanto que cargo público y representante de un partido, está constantemente expuesta al juicio de la audiencia.

\section{Planteamiento del problema y preguntas de investigación}

El objetivo de este trabajo es examinar el papel que juega el humor en un tipo de discurso serio y fuertemente reglado, pero que es también dialéctico en su naturaleza discursiva y argumentativa. Por tanto, en este contexto resulta sumamente interesante explorar tanto los procedimientos como las funciones del humor en la dinámica del debate parlamentario. La hipótesis de trabajo sería en este caso que, si bien el empleo del humor puede suponer ciertos riesgos para la imagen del político, este se muestra a su vez como un instrumento poderoso de argumentación, con el que el emisor puede mostrar la superioridad, a través del ingenio, sobre el adversario.

Las preguntas de investigación que plantea este trabajo pueden esquematizarse en los puntos siguientes:

a) ¿Cuál es el comportamiento del humor en un discurso serio y fuertemente normativizado como el parlamentario?

b) ¿Hasta qué punto el humor, que supone un riesgo para la imagen del que lo emplea, puede poner en peligro la imagen del político?

En este sentido, mi objetivo en este trabajo ha sido analizar las funciones del humor en un discurso serio y sometido a férreas normas de interacción como es el parlamentario, dentro del nivel interactivo o de gestión de imagen. 


\section{Metodología y corpus}

\subsection{Caracterización y delimitación del corpus}

Son numerosos los trabajos sobre humor conversacional que han fijado su análisis en conversaciones reales, todos ellos realizados a partir de transcripciones de grabaciones de audio (Norrick, 1993; Kotthoff, 1996; Holmes, 2000). Sin embargo, para el desarrollo del presente estudio he optado por llevar a cabo un análisis lingüístico basado en la observación de interacciones reales, desviándome de otros enfoques metodológicos como el introspectivo o aquellos basados en corpus informatizados. Asimismo, dichas interacciones reales se encuadran dentro de un contexto per se no humorístico, como es el discurso en la Cámara de diputados. En el caso del humor, esta metodología es particularmente importante, pues no se ha tratado excesivamente: abundan los trabajos basados en el análisis de chistes narrativos (Giora, 1991; Yus, 2008) o monólogos humorísticos (Buxton, 1987), pero son pocos los dedicados a un discurso serio en el cual se deben aislar los enunciados humorísticos, y generalmente, todos ellos se centran en el ámbito de las relaciones laborales entre trabajadores de grandes empresas (Holmes, 2000; Arfeen, 2009). Por lo tanto, la importancia de la metodología empleada en este trabajo reside en su novedad, pues, como digo, los analistas apenas han abordado el estudio del humor en contextos serios y sometidos a unas fuertes reglas de interacción.

El corpus sobre el que he trabajado es de elaboración propia, y está compuesto por 362 documentos escritos en soporte electrónico (archivos .pdf) que recogen la transcripción de las reuniones celebradas en la Asamblea de Madrid durante el periodo que va desde el 1 de enero de 2010 hasta el 31 de diciembre de 2011, ambos inclusive': el denominado Diario de Sesiones, al cual se puede acceder libremente a través de la página web de la Asamblea de Madrid ${ }^{2}$. Mi objetivo ha sido tratar de descubrir de qué manera y hasta qué punto el humor repercute en la imagen de los hablantes, sus compañeros de partido y sus adversarios.

\footnotetext{
${ }^{1}$ Durante este periodo, España, y en especial la Comunidad de Madrid, conoció una situación política bastante particular: la presidencia de la Comunidad Autónoma de Madrid era del Partido Popular (PP), con Esperanza Aguirre a la cabeza, siendo el Partido Socialista (PSOE) el principal grupo de la oposición, con Tomás Gómez Franco como Secretario General. A nivel nacional, en cambio, tenía lugar la situación opuesta: el PSOE, con José Luis Rodríguez Zapatero como líder, era el partido gobernante, mientras que el PP, con Mariano Rajoy al frente, ejercía las labores de principal partido de la oposición. Por ello, en la Asamblea de Madrid se establecieron unas relaciones políticas con el gobierno central bastante tensas, que tuvieron su lógico reflejo en las diferentes intervenciones de los diputados.

2 http://www.asambleamadrid.es/ES/default.aspx
} 
Los criterios que he empleado a la hora de discriminar los enunciados humorísticos de los no humorísticos han sido dos: en primer lugar, la indicación en la transcripción del desencadenamiento de la risa entre los partidarios del hablante; ${ }^{3}$ en segundo lugar, y dado que la risa no es siempre un indicador de la presencia humor, la intención clara del hablante de realizar humor mediante de algún tipo de desviación formal o de contenido con respecto a lo considerado como establecido dentro de un debate parlamentario: una expresión con doble sentido, una exageración formal, una digresión con respecto al tema que se está debatiendo, etc. ${ }^{4}$

\section{Marco teórico}

\subsection{La imagen y el concepto de ethos}

Antes de entrar a hablar específicamente de la imagen política, hemos de considerar el concepto de imagen, tal y como fue definido por Goffman (1987). Esta noción posee una concepción dual: junto a la imagen de carácter individual, o identidad, existe una imagen de naturaleza colectiva, que se configura a partir de la forma en que la identidad se proyecta y gestiona en relación con las identidades de los demás (Hernández Flóres, 2013: 178). La imagen, según Goffman, se manifiesta a partir de tres factores: el contexto, la apariencia y los modales, que son el vehículo a través del cual el individuo adapta la apariencia al contexto; y se va fijando en función de la repetición de dichos modales en distintas situaciones sociales: "La imagen posee un carácter dinámico e interpersonal, que se define en la relación con los demás" (Hidalgo Downing, 2009: 125). ${ }^{5}$

Por lo que respecta específicamente al ámbito político, si atendemos a la bibliografía, a lo largo de los últimos 30 años la comunicación del discurso político ha sido analizada a partir de perspectivas sociopragmáticas ${ }^{6}$. En algunas de ellas, como Charaudeau (2009), juega un papel importante la distinción clásica aristotélica de ethos, logos y pathos, que,

\footnotetext{
${ }^{3}$ Los enunciados del corpus que provocaban risa entre los adversarios del hablante no podían ser considerados humorísticos: se trataba de actos de habla ofensivos, generalmente amenazas o insultos, donde la risa no actuaba como respuesta al enunciado humorístico, sino como mecanismo de mitigación o desprecio de la ofensa.

${ }^{4}$ He elegido estos criterios porque me han parecido lo suficientemente objetivos como para realizar un análisis serio, toda vez que el corpus está basado en textos escritos y, por lo tanto, carentes de cualquier aspecto paraverbal que pueda ayudar a una interpretación más fiel. Es cierto que a lo largo de las transcripciones han aparecido numerosos enunciados que, aunque a mí me resultaban hilarantes, no provocaban las risas entre el auditorio. Por ello, me ha parecido demasiado subjetivo considerar dichos enunciados como humorísticos y, en consecuencia, he preferido apartarlos del análisis.

${ }^{5}$ Para más información sobre la imagen social véase Hernández Flóres (2013), donde se puede encontrar un repaso de este concepto a lo largo de los últimos trabajos de prestigiosos investigadores.

${ }^{6}$ Veánse Alcaide Lara $(2004,2011,2014,2015,2016)$.
} 
a mi juicio, resulta muy válida para caracterizar el discurso político. Según esta perspectiva, en cualquier discurso, y fundamentalmente en el de naturaleza política, vamos a contar con tres factores: el logos, o el discurso en sí; el pathos, o las emociones suscitadas entre el auditorio; y el ethos, o la imagen que el orador proyecta a partir de su discurso, y en la que entran en juego tanto aspectos verbales (la elección de las palabras, el tono, la velocidad de habla...) como extraverbales o contextuales (la vestimenta, la posición del cuerpo, la mirada... $)^{7}$. Los tres guardan una estrecha relación entre sí, de manera que el hablante, a través de su logos, busca proyectar un ethos hacia sus oyentes con el cometido de despertar en ellos un pathos, que, a su vez, contribuya a la conformación de su propio ethos.

Por lo tanto, tanto logos como pathos son aspectos dependientes del ethos, en el sentido de que o bien proceden de este (pathos) o bien lo hacen surgir (logos). Por ello, la forma en que los hablantes primero crean y luego proyectan su ethos ha gozado de gran interés entre los analistas, quienes lo han visto como el núcleo de cualquier discurso que busque, de una manera u otra, persuadir a una determinada audiencia. En este sentido, considero muy acertada la definición que hace del ethos Montero (2012):

Así pues, el ethos se define como la imagen que el orador construye y proyecta de sí mismo en su discurso, imagen que contribuye a asegurar su autoridad, su eficacia y su credibilidad. En otras palabras, se trata del conjunto de rasgos o características que el orador muestra de sí mismo a fin de atraer la atención de su auditorio y de persuadirlo de forma eficaz (Montero, 2012, p. 228).

No obstante, a lo largo de los últimos 20 años algunos autores han modificado en cierta manera el concepto clásico de ethos, ampliando su rango de lo puramente verbal a lo situacional. Maingueneau (2002), por ejemplo, reconoce la existencia de un ethos prediscursivo, que guarda estrecha relación con la noción de imagen. Este ethos prediscursivo es especialmente relevante en el ámbito político, pues en su configuración entran en juego aspectos como la ideología del hablante, sus gustos, aficiones o compañías: El ethos está crucialmente ligado al acto de enunciación, pero no se puede ignorar que el público construye también representaciones del ethos del enunciador antes incluso de que hable. Parece necesario, entonces, establecer una distinción entre ethos discursivo y ethos prediscursivo (Maingueneau, 2002).

\footnotetext{
${ }^{7}$ No obstante, en trabajos como Watts (2002), Locher y Watts (2005), Locher (2008) o Spencer-Oatey (2008), se considera que la imagen es un elemento fluctuante que se va configurando a través del desarrollo del discurso.
} 


\subsection{La imagen politica y el ethos}

A mi juicio, en Charaudeau (2005) encontramos la caracterización más interesante de los distintos ethos que construyen y proyectan los políticos a través de sus discursos. Según este trabajo, el hablante político construye su imagen en función de dos ethos: el ethos de credibilidad y el ethos de identificación. El primero tiene que ver con la capacidad del político de saber hacer, es decir, de su aptitud como personaje político. El segundo, en cambio, guarda relación con la imagen de sí mismo que proyecta hacia los demás; es decir, de su actitud como persona pública.

El ethos de credibilidad depende de tres condiciones comportamentales: la condición de realización, la condición de sinceridad y la condición de eficacia. En virtud de la condición o condiciones que el político satisfaga, proyectará uno u otro de los ethos que a su vez conforman el ethos de credibilidad. Si satisface la condición de realización, el hablante pone el juego el ethos de seriedad, que consiste en ajustar los proyectos a la realidad, en suma, en no frivolizar. Si en cambio, satisface la condición de sinceridad, el ethos que se activa es el de virtud, que consiste en actuar de forma recta, es decir, en mantener una misma línea de pensamiento y acción. La tercera condición que puede satisfacer el político es la condición de eficacia, y en este caso se plantea el ethos de competencia, que consiste en mostrar que se posee la capacidad y el conocimiento necesarios para cumplir los objetivos propuestos con resultados positivos.

Por lo que respecta al ethos de identificación, y debido a que la imagen pública que puede dar un político es muy heterogénea, en él se incluyen todos los ethos relacionados con aspectos físicos y emocionales. En primer lugar, tenemos los ethos de potencia y carácter, que poseen características similares: mientras que el primero se refiere a aspectos puramente físicos, relacionados con conductas como la práctica de un deporte o la movilidad (político como sujeto que actúa), el segundo tiene que ver más con factores anímicos, y en su configuración entran en juego actitudes como la capacidad de crítica, el autocontrol, etc. (político como sujeto que quiere actuar). También, dentro del ethos de identificación encontramos el ethos de inteligencia, relacionado tanto con el capital cultural del hombre político como con la capacidad de salir airoso de las dificultades; el ethos de humanidad, correspondiente tanto con la manifestación de los gustos y las aficiones como con la capacidad de empatizar y encajar las críticas; el ethos de líder, relativo a la capacidad de liderazgo del político; y el ethos de solidaridad, que hace referencia tanto a la voluntad de 
no destacar sobre los demás miembros del partido como a los deseos de escuchar y respetar las opiniones del adversario.

\section{Análisis de los datos}

Por lo que respecta al análisis y clasificación de los enunciados humorísticos desde el punto de vista de la labor de imagen, se ha optado por partir de la clasificación de los distintos tipos de ethos desarrollada en Charaudeau (2005), la cual ha sido expuesta anteriormente. Para cada uno de los ejemplos se han analizado los siguientes aspectos:

a) Los significados explícitos e implícitos que transmite el enunciado en el contexto de la intervención del hablante. La implicatura conversacional que se infiere del enunciado aparece entre llaves (Grice, 2005).

b) La actividad de imagen desarrollada, es decir, sobre qué imagen del destinatario (positiva o negativa) incide la burla, y si existe algún beneficio para esta, en línea con Brown \& Levinson $(1978 / 87)^{8}$. No obstante, hay que tener en cuenta que, en el mundo del discurso político, es una tarea difícil separar la imagen positiva de la negativa. Afectarían a la imagen positiva del político todas aquellas acciones enfocadas a fomentar el derecho del político a ser admirado y reconocido, es decir, todas aquellas características de él mismo y su partido que este quiere transmitir y que, en consecuencia, pretenden ser el foco de atracción del voto de los ciudadanos ${ }^{9}$. En cambio, incidirían sobre la imagen negativa todas aquellas acciones dirigidas a desarticular el derecho del político a actuar libremente, es decir, todas las actitudes críticas que guardasen relación con las acciones políticas efectivas o las líneas de acción propuestas (pactos de gobierno o legislatura, aprobación de leyes, proposición de iniciativas...), en la medida en que los ataques hacia ellas tendrían como objetivo modificarlas, eliminarlas o impedirlas. Evidentemente, en una gran parte de los casos, ambas imágenes pueden verse afectadas, una de forma explícita y la otra de forma implícita, como veremos posteriormente en los análisis.

c) La estrategia de descortesía empleada, en línea con Culpeper (1996). ${ }^{10}$

\footnotetext{
8 Bien es cierto que, a la hora de dar cuenta del fenómeno de la actividad de imagen, la distinción que encontramos en Bravo (1999) entre imagen de afiliación e imagen de autonomía se ha revelado bastante más eficaz que la división tradicional de Brown y Levinson (1978/87). No obstante, el autor del presente artículo considera que la distinción de Bravo (1999) no se adecúa suficientemente a sus propósitos de análisis, toda vez que, como se ha mencionado, este parte de la clasificación de los ethos expuesta en Charaudeau (2005), la cual a su vez toma como base la división clásica entre imagen positiva y negativa.

${ }_{9}^{9}$ Características que, precisamente, coinciden con los componentes del ethos del político.

10 El enfoque de Culpeper (1996) es una reformulación de las ideas sobre cortesía de Brown \& Levinson (1978/87). Él considera que existen cinco tipos de descortesía: una descortesía abierta, que se produce
} 
Asimismo, con el objeto de mostrar más claramente la manera en que la burla humorística incide sobre la imagen del político, cada caso irá encabezado por un ejemplo de ataque a un determinado ethos dentro de un marco serio, a modo de contraste.

\subsection{Ataques al ethos a través del bumor}

4.1.1 Ataques al ethos de credibilidad

Este ethos es el que más ataques ha sufrido dentro del corpus analizado. La razón reside en que el ethos de credibilidad forma parte de la imagen positiva del político y es uno de los pilares del liderazgo: despertar la confianza de los ciudadanos. Que la mayoría de los ataques hayan tenido como objetivo este ethos induce a pensar que el humor que realizan los políticos en las cámaras de diputados tiene como principal diana la faceta política del destinatario. De ello se infiere que el humor parlamentario es una más de las muchas herramientas verbales con las que cuenta el político a la hora de aumentar su imagen y disminuir la del destinatario: un recurso persuasivo de comunicación. Como también se ha señalado, dicho ethos está constituido tres ethos: el de seriedad, el de virtud y el de competencia.

El ejemplo que se expone a continuación está extraído de Fernández García (2009) y es un claro caso de ataque al ethos de seriedad. En él vemos cómo Zapatero acusa abiertamente Rajoy de no tomarse en serio un asunto que afectó gravemente a buena parte de la ciudadanía española, como fue la crisis del Prestige. ${ }^{11}$

Contexto: Primer debate electoral entre José Luis Rodríguez Zapatero y Mariano Rajoy, celebrado el lunes, 25 de febrero de 2008.

Hablante: José Luis Rodríguez Zapatero, líder del Partido Socialista Obrero Español.

cuando "el acto amenazante para la imagen se realiza de manera directa, clara, concisa y sin ambigüedad [...]" (Culpeper, 1996: 356); una descortesía positiva, que se realiza cuando el hablante ataca la imagen positiva de su interlocutor; una descortesía negativa, que tiene lugar cuando el hablante ataca la imagen negativa de su destinatario; una cortesía burlona, que se produce cuando "el acto ofensivo para la imagen se realiza mediante el uso de estrategias de cortesía que son claramente insinceras" [...] (Culpeper, 1996: 256); y una cortesía retenida, que se produce cuando no se da cortesía en circunstancias en que debería haberla.

${ }^{11} \mathrm{La}$ crisis del Prestige fue una crisis ecológica que tuvo lugar en el año 2002 a consecuencia del accidente y posterior hundimiento del petrolero Prestige en aguas del Atlántico, frente a las costas de Galicia. Las 77.000 toneladas de crudo que transportaba el barco provocaron una marea negra que afectó a más de $2.000 \mathrm{~km}$ de costa española y francesa, dando lugar a uno de los mayores desastres medioambientales en la historia de Europa. 
Enunciado: "Cuando había problemas en Galicia del chapapote se iban de caza. Eso solo pertenece a sus gobiernos.” (Fernández García, 2009: 288)

Contrastivamente, a fin de ver cómo actúa el humor sobre la imagen del objeto de la burla, se expone a continuación un caso extraído del corpus elaborado para el presente trabajo. Como en el caso anterior, se trata de un ataque al ethos de seriedad del adversario político:

1. Contexto: Reunión de la Comisión de Presupuestos, Economía y Hacienda, celebrada el miércoles 21 de septiembre de 2011.

Hablante: Sr. Carmona Sancipriano, diputado del Grupo Parlamentario Socialista.

Tema: Información sobre los planes y las acciones futuras de la Consejería de Economía y Hacienda.

Enunciado: "Usted tiene dos buenos Viceconsejeros: una Viceconsejera de gestión y un Viceconsejero anarco-capitalista, que es como se definen algunos de los suyos en los medios de comunicación." (Risas.)

En este caso, el hablante alude irónicamente a la forma en que el viceconsejero de gestión de la Comunidad de Madrid se define a sí mismo. Con ello, realiza una evaluación negativa de este, al que acusa de frivolizar con su cargo público: \{el viceconsejero de gestión no es una persona seria, pues se define a sí mismo ideológicamente de un modo irreal e incoherente con respecto al puesto ocupa\}. Como vemos, emplea una estrategia explícita de descortesía negativa con la que busca un doble propósito: directamente, dañar la imagen de su adversario (y, por extensión, su ethos de seriedad); e indirectamente, la de la Presidenta de la Comunidad de Madrid (y, por extensión, su ethos de competencia), a la que, implícitamente, acusa de rodearse de gente poco seria: \{usted elige a gente que no es seria para configurar su equipo de gobierno . En este sentido, la implicatura podría afectar a la imagen negativa de la presidenta, al poner en duda su capacidad para llevar a cabo acciones políticas competentes.

Con respecto a los ataques vertidos sobre el ethos de virtud dentro de un marco serio, me parece interesante señalar el siguiente ejemplo, extraído Fuentes Rodríguez (2010). En él, el líder del Partido Popular, Mariano Rajoy, acusa directamente al ex presidente José Luis Rodríguez Zapatero de actuar de forma incoherente y de mentir a la ciudadanía sobre el punto de vista de la guerra de Irak que tenía el PSOE: 
Contexto: Primer debate electoral entre José Luis Rodríguez Zapatero y Mariano Rajoy, celebrado el lunes 25 de febrero de 2008 .

Hablante: Mariano Rajoy, líder del Partido Popular.

Enunciado: "En cualquier caso, señor Zapatero, usted mintió a los españoles porque usted apoyó en el Consejo de Seguridad el envío de fuerzas militares [a Irak] después de haberlas retirado para quedar bien internamente y porque creía que le daba votos y apoyos." (Fuentes Rodríguez, 2010)

Como en el caso anterior, a continuación se expone un ejemplo, también extraído del corpus elaborado para este trabajo, en el que el ethos de virtud es atacado a través de mecanismos de creación humorística:

2. Contexto: Reunión de los diputados de la Asamblea de Madrid en Sesión Plenaria, celebrada el jueves 22 de diciembre de 2011.

Hablante: Sr. Manglano Albácar, consejero de Economía y Hacienda.

Tema: Consecuencias de la liberalización de horarios de las grandes superficies (centros comerciales) sobre el pequeño comercio.

Enunciado: "Mire, señoría, a juzgar por la artificiosa longitud de los aplausos de su Grupo Parlamentario y por la lógica de su argumentación, lo que me extraña es que usted no haya expresado sus condolencias por la reciente muerte de un dictador asiático comunista."12 (Aplausos y risas en los escaños del Grupo Parlamentario Popular. - Rumores en los escaños del Grupo Parlamentario Socialista)

En este ejemplo el diputado popular emplea un enunciado irónico y no prototípico para reprochar a los diputados socialistas su deriva hacia posiciones de extrema izquierda y, por tanto, el abandono de la ideología progresista. Puesto que se burla de sus destinatarios al asociarlos con valores antidemocráticos, el hablante emplea una estrategia de descortesía negativa encubierta, mediante la cual daña la imagen negativa de los socialistas y, con ella, su ethos de virtud, como se infiere a partir de la siguiente implicatura: \{Ustedes carecen de principios democráticos, pues con frecuencia suelen acercarse a posiciones políticamente extremas\}.

El ethos de competencia ha sido sobre el que más ataques se han vertido en los ejemplos del corpus analizado. Un ejemplo de ataque al ethos de competencia dentro de un marco serio lo encontramos en Fuentes Rodríguez (2010), expuesto a continuación. En él,

12 Se refiere a Kim Jong-Il, antiguo presidente de Corea del Norte, fallecido pocos días antes de celebrarse esta Sesión Plenaria 
comprobamos cómo Rajoy recrimina abiertamente a Zapatero el no haber desempeñado correctamente su labor de presidente del Gobierno durante su segunda legislatura:

Contexto: Primer debate electoral entre José Luis Rodríguez Zapatero y Mariano Rajoy, celebrado el lunes 25 de febrero de 2008.

Hablantes: Mariano Rajoy, líder del Partido Popular.

Enunciado: "Ha hecho dos cosas, y las dos las ha hecho mal: la estructura de España y los tratos con ETA." (Fuentes Rodríguez, 2010).

$\mathrm{Al}$ igual que en los casos anteriores, expongo a continuación un caso de ataque al ethos de competencia mediante recursos humorísticos, procedente del corpus elaborado para este trabajo:

3. Contexto: Reunión de los diputados de la Asamblea de Madrid en Sesión Plenaria, celebrada el jueves 21 de octubre de 2010.

Hablante: Sr. Peral Guerra, diputado del Grupo Parlamentario Popular.

Tema: Escasa inversión de los presupuestos del estado por parte del Gobierno de la nación en la Comunidad de Madrid.

Enunciado: " $\mathrm{Si}$ analizamos el Ministerio inversor por excelencia, Fomento, nos encontramos con que en 2011 la inversión en Madrid disminuye un 33 por ciento [...], un Ministerio que va a invertir en Madrid en 2011 -siempre que Obama y el chino no lo empeoren con una nueva llamada dominical-13 (Risas en los escaños del Grupo Parlamentario Popular) 68,8 euros por habitante en la media de España.”

En este ejemplo, el diputado popular exagera el estado de cosas con el propósito de burlarse del presidente del gobierno, a quien reprocha el carecer de la iniciativa suficiente para abordar los asuntos de Estado. Se trata, por lo tanto, de una estrategia de descortesía negativa, pues recae sobre la libertad de acción del destinatario y, simultáneamente también, sobre su sub-ethos de competencia: \{Zapatero es un incompetente, son los demás los que tienen que empujarle a actuar y decirle cómo gestionar económicamente España\}

\footnotetext{
13 Esta frase hace referencia a las llamadas que Barak Obama, presidente de los Estados Unidos, y Wen Jiabao, primer ministro de China, le hicieron a Zapatero en plena crisis económica mundial, con el objeto de animarle a que realizara ajustes presupuestarios más duros, a fin de estabilizar el déficit español y evitar que la Zona Euro entrase en recesión.
} 


\subsubsection{Ataques al ethos de identificación}

Este ethos ha sido el que menos ataques ha sufrido en los casos analizados, lo que nos lleva a deducir que, tal y como se ha apuntado en la bibliografía, los políticos son conscientes de que, pese a estar en un contexto de confrontación, tienen que cumplir unas reglas del juego político, por lo que tratan de no enfocar sus burlas sobre aspectos propios del ámbito personal de sus adversarios, con la intención de no resultar antidemocráticos; algo que va muy en la línea de las ideas del comportamiento diplomático, el ethos interaccional y la cortesía como marco regulador del intercambio lingüístico, defendidas por autores como Watts (2002) o Pérez de Ayala (2001).

El ethos de identificación, tal y como he especificado anteriormente, está conformado a su vez por seis ethos: potencia, carácter, liderazgo, solidaridad, humanidad e inteligencia.

Los ethos de potencia y carácter, según se ha apuntado, en realidad son las dos caras de una misma moneda: ambos tienen que ver con la capacidad de actuar del político. Sin embargo, mientras que el primero está relacionado con la fortaleza física, el segundo corresponde a la fortaleza anímica. Ya hemos visto sendos ejemplos de ataque a los ethos de potencia y carácter efectuados dentro de un marco serio en el ejemplo de Fernández García (2009): por un lado, un ataque al ethos de potencia de Rajoy por parte de Zapatero, y, simultáneamente, un ataque al ethos de carácter de este último por parte del primero. El siguiente ejemplo, extraído del corpus elaborado para este trabajo, es una muestra de un ataque al ethos de potencia efectuado dentro de un marco humorístico:

4. Contexto: Reunión de la Comisión de Presupuestos, Economía y Hacienda, celebrada el martes 11 de octubre de 2011.

Hablante: Sr. Oficialdegui Alonso de Celada, diputado del Grupo Parlamentario Popular.

Tema: Informar sobre la política ejercida por la Dirección General de Comercio.

Enunciado: "Llegar a las demagogias de las 24 horas y siete días a la semana, el señor Carmona que nos acompaña hoy aquí y que uno enciende cualquier televisión o cualquier radio y le puede encontrar en horarios de mañana, tarde, noche y madrugada, seguro que no trabaja 24 horas siete días." (Risas.)

En este ejemplo, el señor Oficialdegui exagera la realidad mediante el uso de una hipérbole con la intención de recriminar a su adversario la falta de coherencia entre sus palabras y sus actos. Con ello, ridiculiza a su adversario, dibujándolo como un personaje televisivo más 
que como un político al servicio de los ciudadanos. Se trata, por lo tanto, de un ejemplo de humor de superioridad. En cuanto a la actividad de imagen, el hablante ataca abiertamente la libertad de acción de su destinatario, de manera que estamos ante una estrategia de descortesía negativa: $\{$ El señor Carmona defiende una forma de actuar que ni él mismo sigue\}. Con respecto al ataque al ethos de carácter realizado mediante recursos humorísticos, me parece interesante el siguiente ejemplo, procedente asimismo del corpus elaborado para este trabajo:

5. Contexto: Reunión de la Diputación Permanente, celebrada el miércoles 20 de julio de 2011.

Hablante: Sr. van Halen Acedo, diputado del Grupo Parlamentario Popular.

Tema: Informar sobre las instrucciones para el inicio del curso escolar 2011-2012, que conllevará el despido de 3.000 profesores interinos.

Enunciado: "Yo creo que si ustedes no enredan... Ya han estado los de los sindicatos mayoritarios de enseñanza con don Tomás Gómez y han salido enardecidos a tomar la Bastilla...” (Risas en los escaños del Grupo Parlamentario Popular.)

En este caso, el hablante tiene la intención de recriminar la actitud de Tomás Gómez y los sindicatos de enseñanza, para lo cual emplea dos estrategias verbales. Por un lado, exagera la realidad a través de una hipérbole, deformándola grotescamente. Junto a ella, el hablante también echa mano de una metáfora, que además va en la línea de lo defendido por Lakoff y Johnson (1986) acerca de que en la política son habituales las metáforas relativas a la confrontación. Mediante estos dos recursos, el hablante logra crear un nuevo marco que está fuera de lo democrático, donde queda inscrita la actitud del diputado socialista. Por otra parte, el diputado popular emplea en su enunciado una estrategia de cortesía negativa, con la que busca dañar la libertad de acción de los destinatarios, esto es, su imagen negativa.

Centrándonos en los ataques sobre el ethos de inteligencia dentro de un marco no humorístico, considero relevante el siguiente ejemplo extraído de Fuentes Rodríguez (2010), en donde Rajoy acusa abiertamente a Zapatero de ignorar la historia de España.

Contexto: Primer debate electoral entre José Luis Rodríguez Zapatero y Mariano Rajoy, celebrado el lunes 25 de febrero de 2008.

Hablante: Mariano Rajoy, líder del Partido Popular. 
Enunciado: "Eso de la liberalización del suelo, eso es falso. Usted no se entera. Eso no se ha producido nunca en España. Eso es algo que se le ha ocurrido a usted o que se lo ha inventado. Eso no entró en vigor nunca. Usted no se entera de eso." (Fuentes Rodríguez, 2010).

Con respecto a los ataques a dicho ethos dentro de un entorno humorístico, es significativo el siguiente ejemplo, también extraído del corpus elaborado para este trabajo:

6. Contexto: Reunión de los diputados de la Asamblea de Madrid en Sesión Plenaria, celebrada el jueves 11 de marzo de 2010.

Hablante: Sr. Van-Halen Acedo, diputado del Grupo Parlamentario Popular (PP)

Tema: Declaración de la fiesta de los toros como bien de interés cultural.

Enunciado: "No se inquiete por el vocerío porque, como ha citado usted a Ortega, en esos bancos creen que es Ortega Cano, y era Ortega y Gasset." (Risas y aplausos en los escaños del Grupo Parlamentario Popular. - Protestas en los escaños de la izquierda)

Como vemos, en este ejemplo tenemos a un diputado del PP que ataca el ethos de inteligencia de los diputados de izquierdas. Para ello, echa mano del doble referente del apellido Ortega, y resuelve la ambigüedad semántica incidiendo en el referente más popular, José Ortega Cano, en detrimento del erudito, José Ortega y Gasset. De esta manera, desplaza el marco y logra ridiculizar a los destinatarios de su burla al dibujarlos como individuos fuera de lugar. Con respecto a la estrategia de descortesía empleada, el enunciado daña la imagen positiva de los diputados de izquierdas: el hablante asume una posición alejada con respecto a sus destinatarios, que además queda reforzada por el empleo del demostrativo de distancia media esos, en la estructura marcada esos bancos, frente a la no marcada los diputados de iæquierdas, más acorde con el reglamento de la Asamblea y, por lo tanto, más adecuada al contexto de enunciación.

En lo tocante a los ataques al ethos de liderazgo dentro de un marco serio, pueden servir como ejemplo todos los casos expuestos anteriormente dirigidos a Zapatero o Rajoy, es decir, hacia líderes de formaciones políticas. El que se expone a continuación, procedente del corpus sobre el que se ha trabajado, también es un caso de ataque al ethos de liderazgo del político, pero efectuado dentro de un entorno humorístico. :

7. Contexto: Reunión de los diputados de la Asamblea de Madrid en Sesión Plenaria, celebrada el jueves 13 de octubre de 2011. 
Hablante: Sr. Manglano Albacar, consejero de Economía y Hacienda.

Tema: Implantación del impuesto de patrimonio en la Comunidad de Madrid.

Enunciado: "Empiezo por la forma: un domingo, el candidato ordena al Gobierno a aprobar un Impuesto sobre el Patrimonio. El Gobierno responde: a sus órdenes, Rubalcaba. Rubalcaba dice: ¿cómo? Entonces, el Gobierno dice: perdón; a sus órdenes, señor Rubalcaba." (Risas en los escaños del Grupo Parlamentario Popular.)

En este caso, nuevamente, la intención del hablante es la de dibujar a su adversario como alguien autoritario, y por lo tanto, impropio del sistema democrático, como se infiere a través de la implicatura: \{Rubalcaba dirige el PSOE manu militari, no es un líder democrático y, por lo tanto, no es válido para asumir la presidencia del gobierno español\} Como vemos, el hablante, a través de su burla, daña la capacidad de actuar con libertad de su adversario, de manera que se trata de una estrategia de descortesía negativa. Asimismo, la burla incide sobre los ethos de liderazgo y competencia.

$\mathrm{Al}$ igual que sucede con los ethos de potencia y carácter, los ataques a los ethos de humanidad y solidaridad están relacionados entre sí. No obstante, como ya se expuso anteriormente, el primero va enfocado más hacia la empatía con los ciudadanos, mientras que el segundo se centra sobre todo en la empatía con los compañeros de profesión. En este sentido, todas las críticas que los políticos del PP solían verter contra el famoso "talante" de Zapatero son muestras de ataques al ethos de humanidad dentro de un entorno de enunciación serio. El que se expone a continuación, extraído de Fuentes Rodríguez (2010), discurre en esa línea, y en él vemos cómo Mariano Rajoy se burla de la positividad y el aperturismo de los que hacía gala Zapatero, que considera insinceros.

Contexto: Primer debate cara a cara entre José Luis Rodríguez Zapatero y Mariano Rajoy, celebrado el lunes 25 de febrero de 2008.

Hablantes: Mariano Rajoy, líder del Partido Popular.

Enunciado: "Ningún gobierno ha sembrado en democracia tanta tensión y cizaña mientras hablaba de entendimiento, talante y convivencia." (Fuentes Rodríguez, 2010)

A continuación, por contra, se expone un caso de ataque al ethos de humanidad efectuado dentro de un marco humorístico, extraído también del corpus sobre el que se ha realizado el presente análisis: 
8. Contexto: Reunión de los diputados de la Asamblea de Madrid en Sesión Plenaria, celebrada el jueves 18 de octubre de 2010.

Hablante: Sra. Aboín Aboín, diputada del Grupo Parlamentario Popular.

Tema: Políticas de apoyo a la familia desarrolladas por el gobierno de la CAM

Enunciado: "Porque, ¿saben una cosa, señorías? ¿Saben cuánto destina Zapatero a cada dependiente? Siete euros. ¿Qué dependiente puede vivir con siete euros? ¡Por el amor de Dios!" (Risas)

En este caso encontramos una interrogación retórica con la que el hablante pretende burlarse del ethos de humanidad del presidente Zapatero, al que acusa de no dedicar suficiente dinero a los dependientes españoles y, por lo tanto, de no empatizar con las dificultades ajenas. Al tratarse del presidente del gobierno y secretario general del PSOE, este ataque también supone un ataque a los ethos de competencia y líder: \{Zapatero es un político incompetente y un mal presidente del gobierno porque carece de sentimientos hacia los más necesitados\} Por lo que respecta al efecto humorístico, este es provocado a causa de la realidad absurda pero posible que crea el hablante, algo que se logra gracias a que el humor activa un marco específico donde las asunciones y las normas de la vida cotidiana quedan en suspenso. Con respecto a la labor de imagen, nuevamente se trata de un caso descortés contra la imagen positiva del destinatario, pues el hablante manifiesta una oposición clara hacia la actitud del líder.

Con todo, es importante hacer notar que el ethos de humanidad ha sido el que menos ataques ha sufrido en todos los ejemplos analizados, algo lógico si tenemos en cuenta que tiene que ver con aspectos muy íntimos de la personalidad del político. La razón hay que buscarla en que, en efecto, tal y como consideran numerosos analistas, atacar estos factores va en contra de las normas básicas de comportamiento democrático.

En lo referente a los ataques al ethos de solidaridad, es importante destacar que estos suelen ser más frecuentes que los relativos al ethos de humanidad. La explicación la encontramos en el hecho, mencionado anteriormente, de que este ethos pone su foco en las relaciones intragrupales de los políticos, de manera que los ataques que se realizan sobre él no traspasan nunca los límites del comportamiento aceptado como adecuado dentro de una cámara de diputados. También en Fuentes Rodríguez (2010) encontramos un caso de ataque al ethos de solidaridad de Zapatero por parte de Rajoy, quien recrimina al socialista el haber realizado una alianza que, a su juicio, va contra los principios de la democracia. 
Contexto: Primer debate electoral entre José Luis Rodríguez Zapatero y Mariano Rajoy, celebrado el lunes 25 de febrero de 2008.

Hablantes: Mariano Rajoy, líder del Partido Popular.

Enunciado: "Hablar de discordia, una persona que firma el Pacto del Tinell ${ }^{14}$ cuyo objetivo es echar fuera del mapa político de España a un partido que representa a la mitad de los españoles, es lo más antidemocrático que se ha producido en España." (Fuentes Rodríguez, 2010)

El ejemplo que se expone a continuación, contrariamente, es un caso de ataque al ethos de solidaridad dentro de un entorno humorístico, y también procede del corpus sobre el que se ha realizado el presente análisis:

9. Contexto: Reunión de los diputados de la Asamblea de Madrid en Sesión Plenaria, celebrada el jueves 21 de octubre de 2010.

Hablante: Sr. Peral Guerra, diputado del Grupo Parlamentario Popular.

Tema: Inversiones económicas del Gobierno Central en la Comunidad de Madrid.

Enunciado: "Antes de seguir, señorías [...], no me resisto a preguntar a los diputados socialistas de esta Asamblea: Si le conocíais, ¿por qué nos lo trajisteis?; (Risas.- Aplausos en los escaños del Grupo Parlamentario Popular) si con nueve votos, sólo con nueve votos, nos hubiéramos librado de esa auténtica plaga bíblica de irresponsabilidad, incompetencia y sectarismo autodenominada ZP."15

En este ejemplo tenemos a un diputado popular que realiza una interrogación retórica con el propósito de atacar el ethos de solidaridad de los diputados socialistas presentes en la cámara. En concreto, les recrimina del hecho de que no hicieran nada para evitar la llegada de Zapatero al panorama político español. Asimismo, este ataque al ethos de solidaridad supone simultáneamente un ataque a los ethos de inteligencia y competencia, como inferimos a partir de las implicaturas: \{los políticos socialistas son unos irresponsables, porque han permitido que llegue a la política española un incompetente como Zapatero\}. Se trata, por lo tanto, de una estrategia de cortesía negativa, pues el hablante dirige su burla hacia la libertad de acción de sus adversarios políticos. Por otro lado, el efecto humorístico,

\footnotetext{
${ }^{14}$ El Acuerdo para un Gobierno Catalanista y de Izquierdas en la Generalidad de Cataluña, o Pacto del Tinell, fue un acuerdo de gobierno firmado el 14 de noviembre del año 2003 entre el PSC (Partido Socialista de Cataluña), ERC (Esquerra Republicana de Catalunya) e ICV (Iniciativa per Catalunya Verds). En él se sentaban las bases de lo que sería el futuro Estatuto de Autonomía de Cataluña de 2006 y se añadía una cláusula por la cual los firmantes se comprometían a no efectuar ningún acuerdo con el PP, tanto en territorio catalán como fuera de este.

15 ZP era el alias por el que se conocía popularmente a José Luis Rodríguez Zapatero.
} 
en este caso, se desencadena por la manifestación que hace el hablante de una realidad paradójica, y es el descubrimiento de dicha incongruencia lo que provoca un efecto inesperado entre el auditorio, que emite una respuesta en forma de risa.

\section{Descripción de resultados y discusión}

El análisis ha dado como resultado los datos que se recogen en las siguientes tablas.

Tabla 1. Distribución de los ataques por ethos

\begin{tabular}{|l|l|l|}
\hline Tipo de ethos & Número & Porcentaje \\
\hline Seriedad & 33 & $15,27 \%$ \\
\hline Virtud & 36 & $16,66 \%$ \\
\hline Competencia & 58 & $26,85 \%$ \\
\hline Potencia & 10 & $4,63 \%$ \\
\hline Carácter & 14 & $6,49 \%$ \\
\hline Inteligencia & 15 & $6,95 \%$ \\
\hline Humanidad & 2 & $0,94 \%$ \\
\hline Liderazgo & 23 & $10,64 \%$ \\
\hline Solidaridad & 25 & $11,57 \%$ \\
\hline Total & $\mathbf{2 1 6}$ & $\mathbf{1 0 0}$ \\
\hline
\end{tabular}

Tabla 2. Distribución de los ataques por ethos de credibilidad e identificación

\begin{tabular}{|c|c|c|}
\hline Tipo de ethos & Número & Porcentaje \\
\hline Credibilidad & 127 & $58,78 \%$ \\
\hline Identificación & 89 & $41,22 \%$ \\
\hline Total & $\mathbf{2 1 6}$ & $\mathbf{1 0 0}$ \\
\hline
\end{tabular}

Como vemos, el ethos atacado con más frecuencia es el de credibilidad (58,78\%), dentro del cual destaca el ethos de competencia (26,85\%). El ethos de identificación, por contra, es atacado en menor medida (41,22\%), y dentro de este, destacan sobre todo los ataques a los ethos de solidaridad (11,57\%) y liderazgo (10,64\%). Esto quizás se deba a que los políticos se valen del humor como instrumento de trabajo, en el sentido de que es una de las muchas herramientas verbales de las que echar mano para hacer prevalecer su identidad sobre la de su oponente y, con ello, lograrse el favor de su audiencia: votantes, simpatizantes, partidarios, etc.

Con respecto a la relación entre el humor y la política, se concluye que el humor es uno de los mecanismos con los que los políticos son capaces de persuadir a su audiencia: el político, al emplear el humor, proyecta una imagen o ethos (Charaudeau, 2005) que le 
permite obtener un poder simbólico (Bourdieu, 1992), que le servirá para atraer hacia su postura ideológica al mayor número posible de votantes. No obstante, el humor que emplean los políticos tiene un carácter ambiguo con respecto al ethos del hablante: por un lado, el político, al emplear humor, ensalza su face, al presentarse como una persona carismática, ingeniosa y de mentalidad positiva, y, por lo tanto, susceptible de obtener un poder simbólico con el que persuadir a la audiencia; pero, por otro lado, corre el riesgo de crearse una imagen demasiado frívola, o poco seria, en lo que sería un autoataque al ethos de seriedad. Asimismo, se considera que el humor de los políticos posee un marcado tono endogámico, en el sentido de que está creado por políticos que actúan como portadores de una ideología; tiene como diana la faceta política del adversario y gira en torno a asuntos relativos al ámbito de la política, tales como alianzas electorales, pactos de gobierno, tramitación de presupuestos, etc.

Dentro de los debates parlamentarios el humor actúa como instrumento de persuasión que añade expresividad y capacidad crítica y contraargumentativa, pero sin infringir las normas de cortesía parlamentaria ni alterar el hilo temático del discurso. Dicho de otro modo: el humor le permite al parlamentario desarticular las actitudes o puntos de vista de su adversario, o criticar el funcionamiento de las instituciones democráticas, pero siempre dentro de los límites de lo consensuado como comportamiento adecuado en una cámara de diputados (Watts, 1992), en donde los ataques hacia el adversario político y el respeto a los asuntos a discutir establecidos en el orden del día forman parte del ethos interaccional de los participantes (Spencer-Oatey, 2008). En este sentido, juega un papel muy importante la cortesía, que actúa como mecanismo de control interactivo (Pérez de Ayala, 2001).

En lo tocante a la actividad de imagen (facework), el humor les sirve a los parlamentarios para realizar una operación de auto-valorización de la imagen: mediante el humor, el político se postula como una persona ágil mental y verbalmente, logrando de esta manera, por un lado, manifestar ante los adversarios que forma parte de un grupo cohesionado con el que mantiene lazos de connivencia; y por otro, y en relación con el punto anterior, exhibir su capacidad dialéctica de agresión mitigada a su adversario político. En este sentido, la mayor parte de los enunciados humorísticos han ido dirigidos contra la imagen pública del adversario político, bien en su faceta de cargo público, bien en la de líder o representante de una determinada facción ideológica; por el contrario, han sido minoritarios los casos de ataques hacia la figura del político en tanto que persona privada. 
Del mismo modo, la mayoría de los enunciados han tenido como diana aspectos relativos a la vida política (presupuestos, elecciones, pactos de gobierno, declaraciones públicas...), y han sido escasos los dirigidos a elementos propios de la vida privada, como sentimientos, gustos, aficiones o bagaje cultural.

\section{Referencias}

Alcaide Lara, E. (2004). La ironía, recurso argumentativo en el discurso político. Revista de Filología Hispánica 20 (2), 169-189.

Alcaide Lara, E. (2011). Cuando la oralidad "se despierta": la ironía en el discurso político no parlamentario. Sintaxis y análisis del discurso hablado. Sevilla, España: Universidad de Sevilla, 35-50.

Alcaide Lara, E. (2014). La relación argumentación-(des)cortesía en el discurso persuasivo. Pragmática Sociocultural 8 (2), 223-261. https:/ / doi.org/10.1515/soprag-2014-0008

Alcaide Lara, E. (2015). A vueltas con el empleo del pronombre personal sujeto "yo" en el debate parlamentario. En J. Santana Marrero et al. (eds.) La variación en el español actual. Sevilla, España: Universidad de Sevilla, 17-35.

Alcaide Lara, E. (2016). La metáfora y la metonimia como formas de "mostrar el mundo" en el discurso político. Estrategias argumentativas y discurso politico. Madrid, España: Arco Libros, 273-311.

Archakis, A. y Tsakona, V. (2011). Informal Talk in Formal Settings: Humorous Narratives in Greek Parliamentary Debates. En Tsakona, V. y Popa, D. (Eds.) Studies in Political Humor: In Between Political Critique and Public Entertainment. Ámsterdam, Países Bajos: John Benjamins, 61-81. https://doi.org/10.1075/dapsac.46.06arc

Arfeen, B. (2009). Humor in the Workplace: How it's Work and What if Doesn't?. LCOM Papers 1, 1-15.

Atkinson, M. (1988). Our Masters' Voices. Londres, Reino Unido: Routledge.

Attardo, S. (1994). Linguistic Theories of Humor. Berlín, Alemania: Mouton de Gruyter.

Bañón Hernández, A. et al. (2012). La gestión del tiempo en el discurso parlamentario. Discurso \& Sociedad 6 (1), 49-78.

Beattie, G. (1982). Turn-Taking and Interruption in Political Interview: Margaret Thatcher and Jim Callaghan Compared and Contrasted. Semiotica 39 (1-2), 93-103. https://doi.org/10.1515/semi.1982.39.1-2.93

Blas Arroyo, J. L. (2001). 'No diga chorradas...' La descortesía en el debate político cara a cara. Oralia 4, 9-45.

Bourdieu, P. (1992). Language and Symbolic Power. Cambridge, Reino Unido: Polity Press.

Bravo, D. (1999). ¿Imagen positiva vs. imagen negativa?: pragmática socio-cultural y componentes de face. Oralia 2, 155-184.

Brown, P. \& Levinson, S. (1978/1987). Politeness. Some Universals in Language Usage. Cambridge, Reino Unido: Cambridge University Press.

Buxton, R. (1987). The Late-Night Talk Show: Humor in Fringe Television. Southern Speech Communication Journal 52 (4), 377-389. https://doi.org/10.1080/10417948709372704

Charaudeau, P. (2005). Le discours politique: les masques du pouvoir. París, Francia : Vuibert.

Charaudeau, Patrick (2009). La argumentación persuasiva. El ejemplo del discurso político. En M. Shiro et al. (eds.) Haciendo discurso. Caracas, Venezuela: Facultad de Humanidades y Educación de la Universidad Central de Venezuela, 277-295. 
Charaudeau, P (2013). De l'ironie à l'absurde et des catégories aux effets. En D. Vivero García (Ed.), Frontières de l'bumour. París: L'Harmattan, 13-26.

Chilton, P. (2004). Analysing Political Discourse: Theory and practice. Londres: Reino Unido: Routledge.

Connolly, W. (1993). The Terms of Political Discourse. Princeton, Estados Unidos: Princeton University Press.

Cortés Rodríguez, L. (2011). El plano secuencial en los debates en torno al estado de la nación. I. El discurso del presidente. Círculo de Lingüistica Aplicada a la Comunicación 46, 3-50. https://doi.org/10.5209/rev CLAC.2011.v46.1

Cortés Rodríguez, Luis (2015). Análisis del discurso político. Consideraciones acerca de los debates en torno al estado de la nación. Almería, España: Universidad de Almería.

Culpeper, J. (1996). Towards an Anatomy of Impoliteness. Journal of Pragmatics 25, 349-367. https://doi.org/10.1016/0378-2166(95)00014-3

Fernández García, F. (2009). (Des)cortesía y pugna dialéctica en el debate político-electoral. Oralia 12, 267-305.

Fuentes Rodríguez, C. (2010, diciembre). El debate entre Zapatero y Rajoy: Estudio argumentativo. Tonos Digital 20. Recuperado de http://www.tonosdigital.es/ojs/index.php/tonos/article/view/592.

Giora, R. (1991). On the Cognitive Aspects of the Joke. Journal of Pragmatics 16, 465-485. https://doi.org/10.1016/0378-2166(91)90137-M

Goffman, E. (1987). La presentación de la persona en la vida cotidiana. Madrid, España: Murguía.

González Sanz, M. (2014). Tertulia politica y descortesía verbal. Sevilla, España: Facultad de Filología de la Universidad de Sevilla.

Hernández Flores, N. (2013). Actividad de imagen: caracterización y tipología en la interacción comunicativa. Pragmática Sociocultural 1 (2), 175-198.

Holmes, J. (2000). Politeness, Power and Provocation: How Humour Functions in the Workplace. Discourse Studies. 2 (2), 159-185.

https://doi.org/10.1177/1461445600002002002

Hidalgo Downing, R. (2009). Políticos y ciudadanos: análisis conversacional de la entrevista política. Revista Electrónica de Lingüistica Aplicada 8, 89-101.

Hidalgo Downing, R. y Nieto y Otero, M. J. (2014). Estrategias de vinculación afectiva y entonación en un debate político. Oralia 17, 200-235.

Kotthoff, H. (1996). Impoliteness and Conversational Joking: On Relational Politics. Folia Linguistica 30 (3-4), 299-326. https://doi.org/10.1515/flin.1996.30.3-4.299

Lakoff, G. y Johnson, M. (1986). Metáforas de la vida cotidiana. Madrid, España: Cátedra, Teorema.

Lakoff, G. (2007). No pienses en un elefante. Madrid, España: Editorial Complutense.

Lakoff, R. (1990). Talking Power. Nueva York, Estados Unidos: Basic Books.

Locher, M. A. (2008). Relational Work, Politeness and Identity Construction. En G. Antos et al. (Eds.) Handbooks of Applied Linguistics. Vol. 2: Interpersonal Communication. Berlín, Alemania: Mouton de Gruyter, 509-540.

Locher, M. A. y Watts, R. (2005). Politeness Theory and Relational Work. Journal of Politeness Research 1 (1), 9-33. https://doi.org/10.1515/jplr.2005.1.1.9

Maingueneau, D. (2002). Problèmes d'ethos. Pratiques 113-114, 55-67. Traducción en castellano en https:// es.scribd.com/doc/15238597/Ethos-Maingueneau.

Montero, A. S. (2012). Los usos del ethos. Abordajes discursivos, sociológicos y políticos. Rétor 2 (2), 223-242.

Norrick, N. (1993). Conversational Joking: Humor in Everyday Talk. Indiana, Estados Unidos: Indiana University Press. 
Pérez de Ayala, S. (2001). FTAs and Erskine May: Conflicting Needs? - Politeness in Question Time. Journal of Pragmatics 33, 143-169. https://doi.org/10.1016/S03782166(00)00002-3

Romero Nieto, A. (2017). La interrupción con valor humorístico como mecanismo de gestión del poder en el debate parlamentario. Revista Española de Lingüistica 47 (1), 109-132.

Romero Nieto, A. (2018). Prosodia y gestualidad y su relación con la expresión del humor en el debate parlamentario. Cuadernos de Investigación Filológica 44.

https://doi.org/10.18172/cif.3413

Saftoiu, R. y Popescu, C. (2014). Humor as Branding Strategy in Political Discourse. A Case Study from Romania. Signos 47 (85), 293-320.

Spencer-Oatey, H. (2008). Face, (Im)politeness and Rapport. En Spencer-Oatey, H. (Ed.) Culturally Speaking: Culture, Communication and Politeness Theory. Londres, Reino Unido: Continuum, 11-47.

Tsakona, V. (2009). Humor and Image Politics in Parliamentary Discourse: a Greek Case Study. Text \& Talk 29 (2), 219-237.

Tsakona, V. (2013). Parliamentary Punning: Is the Opposition More Humorous than the Ruling Party? European Journal of Humour Research 1 (2), 101-111. https://doi.org/10.1515/TEXT.2009.010

Ruiz Gurillo, L. (2012). La lingüística del humor en español. Madrid, España: Arco/Libros.

Watts, R. (2002). Politeness. Oxford, Reino Unido: Oxford University Press.

Wilson, J. (1990). Politically Speaking. Oxford, Reino Unido: Basil Blackwell.

Yus, F. (2003). Humor and the Search for Relevance. Journal of Pragmatics 35 (9), 1295-1331. https://doi.org/10.1016/S0378-2166(02)00179-0

Yus, F. (2008). A Relevance-Theoretic Classification of Jokes. Lodz Papers in Pragmatics 4 (1), 131-157. https://doi.org/10.2478/v10016-008-0004-4

Alejandro Romero Nieto es licenciado en Filología Hispánica y doctor en Lengua Española por la Universidad Complutense de Madrid. Su trayectoria investigadora se centra en la pragmática y el análisis del discurso, más concretamente en los ámbitos del humor, la cortesía y el discurso político. Ha publicado trabajos en revistas especializadas y compagina la labor investigadora con la impartición de seminarios sobre comunicación política, cortesía y labor de imagen. 